\title{
Late ventricular potentials and heavy drinking
}

\author{
G Pochmalicki, M Genest, H Jibril
}

\begin{abstract}
Objectives-To assess the effects of chronic drinking on detection of low amplitude signals, and to determine the relation between late ventricular potentials (LVP) and liver biopsy findings.

Design-Prospective study. Setting-General hospital.
\end{abstract}

Patients-41 consecutive chronic alcoholics without known pre-existing heart disease.

Methods-About four days after each patient's last alcoholic drink, ECG, echocardiography, signal averaged electrocardiogram, liver biopsy, and blood tests were performed.

Results-Twenty eight per cent of patients had evidence of LVP. There was a correlation between the percentage of steatosis of the hepatic biopsy and the amplitude of the last $40 \mathrm{~ms}$ of average QRS ( $P=0.04)$, the duration of the terminal low amplitude QRS signal $(P=$ $0.05)$, and the number of positive criteria of late potentials $(P=0.02)$.

Conclusions-Chronic drinking sufficient to cause steatosis is associated with positive findings on the signal averaged ECG.

(Heart 1997;78:163-165)

Keywords: alcoholism; late ventricular potentials; signal averaged electrocardiogram

Although population studies ${ }^{12}$ show disparate relations between alcohol intake and causes of death with a "J" shaped curve, cardiovascular death is the most important cause of mortality in alcoholics. ${ }^{3}{ }^{4}$ Indeed, the ascending part of the $\mathrm{J}$ shaped curve could be explained in part by acute heavy ethanol drinking effects on the incidence of arrhythmias, ${ }^{2}$ cardiomyopathy, hypertension, and perhaps silent myocardial ischaemia. ${ }^{5-7}$ In the British Regional Heart Study, Wannamethee and Shaper ${ }^{3}$ found a positive association between heavy drinking (more than six drinks daily) and the risk of sudden death, clearly seen in patients with no evidence of pre-existing ischaemic heart disease. In drinkers who die suddenly, histopathological studies of the liver often show fatty changes ${ }^{89}$ and fatty infiltration of the heart. ${ }^{10}$ To our knowledge, little is known about the effects of drinking on detection of low amplitude signals, which are independent markers of ventricular arrhythmias ${ }^{11}$ and risk of sudden death. In addition, their correlation with fatty infiltration on liver biopsy has not previously been reported in chronic alcoholics. ${ }^{12}$

\section{Patients and methods}

Patients studied were 41 consecutive chronic alcoholics admitted in the alcoholism unit of our hospital over a two year period for liver biopsy (transcutaneous 53\%, transvenous $47 \%$ ), and blood tests of electrolytes and liver enzymes. The liver biopsy specimens were immediately fixed in formaldehyde and embedded in paraffin. Conventionally stained (Masson's trichrome, Perls, haematoxylin and eosin) $4 \mu \mathrm{m}$ sections were used for histopathological evaluation by a liver pathologist, according to well established criteria. The lipid content in the liver biopsies was expressed as the volume fraction of the total parenchyma occupied by fat.

About four days after each patient's last alcoholic drink (to avoid the acute effects of ethanol on the cardiovascular system) several cardiovascular investigations were performed: a 12-lead ECG; bidimensional echocardiography using an Apogee CX 200 (ATL, Washington, USA) with a $3.5 \mathrm{MHz}$ phased array probe to determine (from the parasternal long and short axis views) left atrial diameter, diastolic and systolic left ventricular diameter, and mean fractional shortening (\%);24 hour Holter monitoring (Hewlett Packard New Wave 3.0; Hewlett Packard, Palo Alto, USA); and signal averaged electrocardiogram (Marquette, MacVu; band pass filter range of 40-250 Hz; Marquette, Milwaukee, USA) with automated measurement of the filtered QRS duration, the root mean square (RMS) voltage of the last $40 \mathrm{~ms}$ of the filtered QRS complex, and the duration of the terminal low amplitude QRS signal $<40 \mu \mathrm{V}$ (LAS). Late potentials were diagnosed in the presence of at least two of the following measures: duration of the filtered QRS > $120 \mathrm{~ms}$, RMS voltage of the terminal $40 \mathrm{~ms}$ of the filtered QRS $<20 \mu \mathrm{V}$, and duration of the low amplitude $(<40 \mu \mathrm{V})$ signals of terminal filtered QRS $>40 \mathrm{~ms}$.

STATISTICAL ANALYSIS

Data are expressed as means (SD). Dichotomous variables were compared by $\chi^{2}$ analysis. Continuous variables were compared using a non-parametric Mann-Whitney test. Steatosis and LVP were related to other variables by means of Spearman rank correlation test. The level of significance was $P<0.05$. 


\section{Results}

We studied 41 patients (32 men, and nine women, mean age $49 \cdot 7$ years). Main clinical features, echocardiography results, Holter findings, biopsy results, and serum biochemical values are shown in the table. The mean (SD) reported daily intake of ethanol was 89 (31) $\mathrm{g}$ over a period of 14 (9) years. Twenty eight per cent of patients had evidence of LVP. The mean filtered QRS duration was 106 (12) ms, RMS was 25.6 (11) $\mu \mathrm{V}$, and LAS was 32 (12) ms. No major arrhythmias were recorded by Holter monitoring. The main echocardiographic parameters were normal, so were serum potassium and magnesium concentrations. On the liver biopsy, $80 \%$ of patients had steatosis, $32 \%$ cirrhosis, and $70 \%$ fibrosis.

There was a positive association between the daily ingested quantity of alcohol before the study and the filtered QRS duration ( $P=$ $0.02, r=0.31)$. Moreover, there was a correlation between the percentage of steatosis of the hepatic biopsy and the amplitude of the last $40 \mathrm{~ms}$ of average QRS $(P=0.04, r=$ -0.32 ), the duration of the terminal low amplitude QRS signal ( $P=0.05, r=0.30$ ), and the number of positive criteria of late potentials $(P=0.02, r=0.38)$. Fatty content of the liver was less important in patients without LVP (19\% $v 35 \%, \mathrm{P}=0.02)$. LVP was closely correlated to the serum concentrations of $\gamma$ glutamyl transferase $(P=0.02)$, aspartate aminotransferase $(P=0.04)$, and alanine aminotransferase $(P=0.03)$.

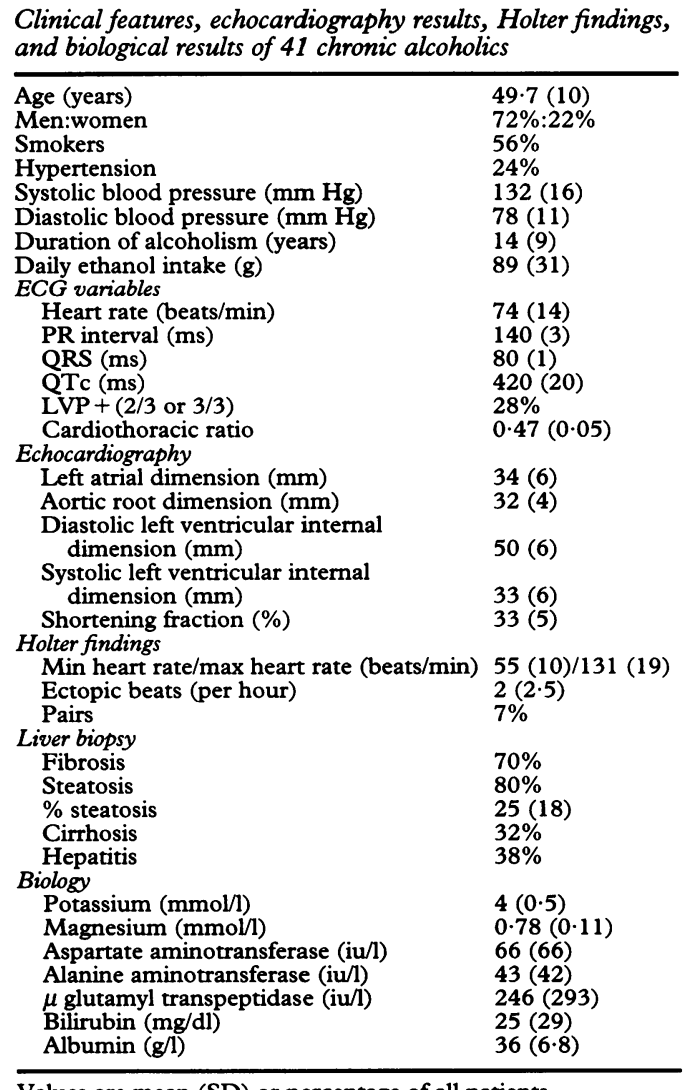

Values are mean (SD) or percentage of all patients.

\section{Discussion}

Disparities in the relations between alcohol consumption and various cardiovascular conditions ${ }^{13}$ are now evident: an inverse relation of alcohol use to coronary artery disease is supported by many population studies, and the data do not suggest monotonic relationships. Light to moderate alcohol intake is associated with a reduction in overall mortality resulting primarily from a reduced risk of coronary artery disease. The level of alcohol consumption is a crucial consideration: progressively greater levels of consumption (at least four units of alcohol per day) are associated with progressively higher all cause mortality. In heavy drinkers, Wannamethee and Shaper ${ }^{3}$ found a twofold increase in risk of sudden death, clearly seen in patients with no evidence of pre-existing heart disease. In patients with alcoholic liver disease, QT interval prolongation was associated with an adverse prognosis. ${ }^{4}$ Heavy drinking can induce cardiac arrhythmias, including ventricular tachycardia, which are associated with sudden cardiac death. Other epidemiological studies have shown an increased incidence of sudden death in alcoholics. Cardiovascular death is an important cause of mortality in alcoholics, and the occurrence of sudden death in alcoholics with cirrhosis or fatty liver probably reflects an increased susceptibility to fatal arrhythmias.

This is consistent with our results also showing that chronic drinking sufficient to cause steatosis is associated with positive findings on signal averaged ECG, which suggests a correlation between cardiac and liver diseases.

LVP have been identified as a prognostic factor in the prediction of ventricular arrhythmias, ${ }^{9}$ especially in postmyocardial infarction patients. They are thought to represent delayed activation of some areas of a diseased myocardium and are therefore considered a marker of an arrhythmogenic substrate. In alcoholic fatty liver, accumulation of fat results from impaired fatty acid oxidation, increased uptake, esterification of fatty acids to triglycerides, and diminished lipoprotein biosynthesis and secretion. ${ }^{14}{ }^{15} \mathrm{~A}$ similar pathway may cause myocardial damage ${ }^{16}$ in drinkers who die suddenly.

\section{CONCLUSIONS}

This study suggests a relation between the risk of ventricular arrhythmias and derangements of hepatic metabolism that could be explained by a common pathogenetic mechanism at the cellular level of ethanol. Chronic alcohol intake sufficient to cause histologically significant fatty liver is associated with LVP. Because it is a simple and non-invasive technique, the detection of LVP could reveal early, preclinical myocardial lesions, and perhaps help to identify alcoholic patients at high risk of lethal arrhythmias.

1 Doll R, Peto R, Hall E, Wheatley K, Gray R. Mortality in relation to consumption of alcohol: 13 years' observain relation to consumption of alcohol: 13 years' observa-
tions on male British doctors. $B M \mathcal{F} 1994 ; 309: 911-18$. 2 Suhonen O, Aromaa A, Reunanen A, Knekt P. Alcohol consumption and sudden coronary death in middle aged consumption and sudden coronary death in middle

3 Wannamethee G, Shaper AG. Alcohol and sudden cardiac 
death. Br Heart J 1992;68:443-8

4 Day CP, James OFW, Butler TJ, Campbell RWF. QT prolongation and sudden cardiac death in patients with alcoholic liver disease. Lancet 1993;341:1423-8.

5 Klatsky AL, Armstrong MA, Friedman GD. Alcohol and mortality. Ann Intern Med 1992;117:646-54.

6 Klatsky AL. Alcohol, coronary disease, and hypertension. Annu Rev Med 1996;47:149-60.

7 Rossinen J, Partanen J, Koskinen P, Toivonen L, Kupari $M$, Nieminen MS. Acute heavy alcohol intake increases silent myocardial ischemia in patients with stable angina pectoris. Heart 1996;75:563-7.

8 Rosmorduc O, Richardet JP, Lageron A, Munz C, Callard $P$, Beaugrand $M$. La steatose hepatique massive: une cause de deces brutal chez le malade alcoolique. Gause de deces brutal chez le mal

9 Copeland AR. Sudden death in the alcoholic. Forensic Sci Int 1985;29:159-69.

10 Vikhert AM, Tsiplenkova VG, Cherpachenko NM. Alcoholic cardiomyopathy and sudden cardiac death. $\dot{f}$ Am Coll Cardiol 1986;8(suppl A):3A-11A.

11 Breidthardt G, Cain ME, El-Sherif N, Flowers NC,
Hombach V, Janse M, et al. Standards for analysis of ventricular late potentials using high resolution or signal average electrocardiography. A statement by a task force committee of the European Society of Cardiology, the American Heart Association, and the American College of Cardiology. 7 Am Coll Cardiol 1991;17:999-1006.

12 Estruch R, Fernandez Sola J, Sacanella E, Paré C, Rubin E, Urbano-Marquez A. Relationship between cardiomyopathy and liver disease in chronic alcoholism. Hepatology 1995;22:532-8.

13 Shaper AG, Wannamethee G, Walker M. Alcohol and mortality in British men: explaining the U-shaped curve. Lancet 1988;i:1267-73. 14 Diamcet $\mathrm{Y}$. Al

Eardiomyopathy. $N$ Engl F Med 1989;320:458-9.

15 Fernandez-Sola J, Estruch R, Grau JM, Paré JC, Rubin E, Urbano-Marquez A. The relation of alcoholic myopathy to cardiomyopathy. Ann Intern Med 1994;120:529-36.

16 Tsiplenkova VG, Vickert AM, Cherpachenko NM. Ultrastructural and histochemical observations in human and experimental alcohol cardiomyopathy. $\mathcal{f} \mathrm{Am}$ Coll Cardiol 1986;8:22A-32A 